This is a self-archived - parallel published version of this article in the publication archive of the University of Vaasa. It might differ from the original.

\title{
Spectral Decompositions of Selfadjoint Relations in Pontryagin Spaces and Factorizations of Generalized Nevanlinna Functions
}

Author(s): Hassi, Seppo; Wietsma, Hendrik Luit

Title: $\quad$ Spectral Decompositions of Selfadjoint Relations in Pontryagin Spaces and Factorizations of Generalized Nevanlinna Functions

Year: $\quad 2020$

Version: Published version

Copyright C Springer Nature Switzerland AG 2020.

\section{Please cite the original version:}

Hassi S. \& Wietsma H.L. (2020). Spectral Decompositions of Selfadjoint Relations in Pontryagin Spaces and Factorizations of Generalized Nevanlinna Functions. In: Alpay D., Fritzsche B., Kirstein B. (eds.) Complex Function Theory, Operator Theory, Schur Analysis and Systems Theory : A Volume in Honor of V.E. Katsnelson, 515-534. Operator Theory: Advances and Applications, 280. Cham: Birkhäuser. https://doi.org/10.1007/978-3-030-44819-6_16 


\title{
Spectral decompositions of selfadjoint relations in Pontryagin spaces and factorizations of gen- eralized Nevanlinna functions
}

\author{
Seppo Hassi \& Hendrik Luit Wietsma \\ Dedicated to V.E. Katsnelson on the occasion of his 75th birthday
}

\begin{abstract}
Selfadjoint relations in Pontryagin spaces do not possess a spectral family completely characterizing them in the way that is known to hold for selfadjoint relations in Hilbert spaces. Here it is shown that a combination of a factorization of generalized Nevanlinna functions with the standard spectral family of selfadjoint relations in Hilbert spaces can function as a spectral family for selfadjoint relations in Pontryagin spaces. By this technique additive decompositions are established for generalized Nevanlinna functions and selfadjoint relations in Pontryagin spaces.
\end{abstract}

Mathematics Subject Classification (2000). Primary: 47B50; Secondary: 46C20, 47A10, 47A15.

Keywords. Generalized Nevanlinna functions, selfadjoint (multi-valued) operators, (minimal) realizations.

\section{Introduction}

It is well known that the class of generalized Nevanlinna functions can be realized by means of selfadjoint relations in Pontryagin spaces (cf. Section 2.2 below). In [16] it has been shown that there is a strong connection between the factorization result for scalar generalized Nevanlinna functions and the invariant subspace properties of selfadjoint relations in Pontryagin spaces. Here that approach is extended to the case of operator-valued generalized Nevanlinna functions whose values are bounded operators on a Hilbert space $\mathcal{H}$; in what follows this class is denoted by $\mathfrak{N}_{\kappa}(\mathcal{H})$, where $\kappa \in \mathbb{N}$ refers to the number of negative squares of the associated Nevanlinna kernel; see [11, 12]. More precisely, by combining the multiplicative factorization for operator-valued generalized Nevanlinna functions established in [14] with the 
well-known spectral family results for selfadjoint operators in Hilbert spaces the following additive decomposition is obtained.

Theorem 1.1. Let $F \in \mathfrak{N}_{\kappa}(\mathcal{H})$ and let $\Delta$ be a measurable subset of $\mathbb{R} \cup\{\infty\}$ or a closed symmetric subset of $\mathbb{C} \backslash \mathbb{R}$. Then $F$ can be written as $F_{\Delta}+F_{R}$, where

(i) $\sigma\left(F_{\Delta}\right) \subseteq \operatorname{clos} \Delta$ and int $\Delta \subseteq \rho\left(F_{R}\right)$;

(ii) $F_{\Delta} \in \mathfrak{N}_{\kappa_{\Delta}}(\mathcal{H}), F_{R} \in \mathfrak{N}_{\kappa_{R}}(\mathcal{H})$ and $\kappa_{\Delta}+\kappa_{R} \geq \kappa$.

If $\partial \Delta \cap \operatorname{GPNT}(F)=(\operatorname{clos}(\Delta) \backslash \operatorname{int}(\Delta)) \cap \operatorname{GPNT}(F)=\emptyset$, then the decomposition may be chosen such that $F_{\Delta}$ and $F_{R}$ do not have a generalized pole in common. In this case, $\kappa_{\Delta}+\kappa_{R}=\kappa$.

In Theorem $1.1 \rho(F)$ denotes the set of holomorphy of $F \in \mathfrak{N}_{\kappa}(\mathcal{H})$ in $\mathbb{C} \cup\{\infty\}$ and $\sigma(F)$ stands for its complement in $\mathbb{C} \cup\{\infty\}$. For the definition of generalized poles and generalized poles not of positive type (GPNTs), see Section 2.2 below. It should be mentioned that Theorem 1.1 generalizes a result obtained for matrixvalued generalized Nevanlinna functions by K. Daho and H. Langer in [2, Prop. $3.3]$.

For the proof of Theorem 1.1 spectral families for Pontryagin space selfadjoint relations are replaced by factorizations of generalized Nevanlinna functions in combination with the standard spectral decompositions of selfadjoint Hilbert space operators (or relations); this is the main contribution of this paper. Such an approach is needed because spectral families for Pontryagin space selfadjoint relations do not exist in an appropriate form to establish Theorem 1.1; cf. [13]. This approach can be extended to decompose for instance definitizable functions (and operators) in a Kreı̆n space setting. Starting from the essentially multiplicative representation of an definitizable function $F$ in [10, Thm. 3.6] one can for example show that $F$ can be written as the sum of two definitizable functions $F_{+}$and $F_{-}$, where $F_{+}$has no points of negative type and $F_{-}$has no points of positive type.

The intimate connection between generalized Nevanlinna functions and selfadjoint relations in Pontryagin spaces, see e.g. Section 2.2 below, means that the following analogue of Theorem 1.1 holds for selfadjoint relations in Pontryagin spaces. For the notation $\operatorname{ENT}(A)$ in the following theorem, see Section 2.1 below.

Theorem 1.2. Let $A$ be a selfadjoint relation in a Pontryagin space $\{\Pi,[\cdot, \cdot]\}$ with $\rho(A) \neq \emptyset$ and let $\Delta$ be either a measurable subset of $\mathbb{R} \cup\{\infty\}$ or a closed symmetric subset of $\mathbb{C} \backslash \mathbb{R}$. Then there exists a selfadjoint relation $A_{e}$ in a Pontryagin space $\left\{\Pi_{e},[\cdot, \cdot]_{e}\right\}$ with $\operatorname{gr}(A) \subseteq \operatorname{gr}\left(A_{e}\right)$ and a decomposition $\Pi_{\Delta}[+] \Pi_{R}$ of $\Pi_{e}$ such that

(i) $\left\{\Pi_{\Delta},[\cdot, \cdot]\right\}$ and $\left\{\Pi_{R},[\cdot, \cdot]\right\}$ are Pontryagin spaces;

(ii) $\Pi_{\Delta}$ and $\Pi_{R}$ are $A_{e}$-invariant;

(iii) $\sigma\left(A_{e}\left\lceil_{\Pi_{\Delta}}\right) \subseteq \operatorname{clos} \Delta\right.$ and int $\Delta \subseteq \rho\left(A_{e}\left\lceil_{\Pi_{R}}\right)\right.$.

If $\partial \Delta \cap \operatorname{ENT}(A)=\emptyset$, then $A_{e}$ and $\Pi_{e}$ can be taken to be $A$ and $\Pi$, respectively, and the decomposition can be taken such that

$$
\sigma_{p}\left(A{\Pi_{\Delta}}\right) \cap \sigma_{p}\left(A\left\lceil_{R}\right)=\emptyset .\right.
$$


In the particular case that $\Delta$ is a closed symmetric subset of $\mathbb{C} \backslash \mathbb{R}$ the decomposition in Theorem 1.2 is directly obtained by means of Riesz projection operators; see e.g. [1, Ch. 2: Thm 2.20 \& Cor. 3.12]. However, Theorem 1.2 cannot always be established by means of spectral families of selfadjoint relations in Pontryagin spaces if $\partial \Delta \cap \operatorname{ENT}(A) \neq \emptyset$. Indeed the eigenspaces of ENTs can be neutral or even degenerate; in such cases the corresponding eigenvalues are critical points and the spectral family might not be extendable to sets having these points as their endpoints; cf. [13, Comments following Thm. 5.7].

To mention another example of decompositions included in Theorem 1.2 consider $\Delta=(-\infty, a) \cup(b, \infty) \cup\{\infty\}$, where $a, b \in \mathbb{R} \backslash \operatorname{ENT}(A)$ and $a<b$. Then Theorem 1.2 says that a selfadjoint relation in a Pontryagin spaces can be decomposed into an unbounded selfadjoint relation in a Pontryagin space and a bounded selfadjoint operator in a Pontryagin space; for selfadjoint operators this last result can be found in [11]; see also the references therein. Note that intervals $\Delta$ of the given type naturally arise in connection with rational functions; for instance when considering definitizable operators or the products of (generalized) Nevanlinna functions with rational functions, see e.g. [8].

Finally the contents of the paper are shortly outlined. The first half of Section 2 consists of an introduction to selfadjoint relations (multi-valued operators) in Pontryagin spaces together with a short overview of minimal operator realizations of (operator-valued) generalized Nevanlinna functions. In the latter half of this section we recall some results about how non-minimal realizations can be reduced to minimal ones and also consider the (minimality of the) realization for the sum of generalized Nevanlinna functions. In Section 3 we first establish the connection between a factorization of a generalized Nevanlinna function and the spectral properties of its operator realization. This result is a key tool for using the factorization of generalized Nevanlinna functions as a replacement for a spectral decomposition of selfadjoint relations in Pontryagin spaces. Finally, in the second and third subsections of Section 3 Theorems 1.1 and 1.2 are proven, respectively.

\section{Preliminaries}

The first two subsections contain introductions to (unbounded) operators or more generally linear relations in Pontryagin spaces and (minimal) operator realizations for generalized Nevanlinna functions, respectively. In the third subsection it is shown how non-minimal realizations may be reduced to minimal ones. Finally, in the fourth subsection the sum of generalized Nevanlinna functions is considered.

\subsection{Linear relations in Pontryagin spaces}

A linear space $\Pi$ together with a sesqui-linear form $[\cdot, \cdot]$ defined on it, is a Pontryagin space if there exists an orthogonal decomposition $\Pi^{+}+\Pi^{-}$of $\Pi$ such that $\left\{\Pi^{+},[\cdot, \cdot\}\right.$ and $\left\{\Pi^{-},-[\cdot, \cdot]\right\}$ are Hilbert spaces either of which is finite dimensional; 
here orthogonal means that $\left[f^{+}, f^{-}\right]=0$ for all $f^{+} \in \Pi^{+}$and $f^{-} \in \Pi^{-}$. For our purposes it suffices to consider only Pontryagin spaces for which $\Pi^{-}$is finitedimensional; its dimension (which is independent of the orthogonal decomposition $\left.\Pi^{+}+\Pi^{-}\right)$is the negative index of $\Pi$.

A (linear) relation $H$ in $\{\Pi,[\cdot, \cdot]\}$ is a multi-valued (linear) operator whose domain is a linear subspace of $\Pi$, denoted by $\operatorname{dom} H$, and which linearly maps each element $x \in \operatorname{dom} H$ to a subset $H x:=H(x)$ of $\Pi$. (Graphs of) linear relations on $\Pi$ can be identified with subspaces of $\Pi \times \Pi$; in what follows this identification will tacitly be used. The linear subspace $H(0)$ is called the multi-valued part of $H$ and is denoted by mul $H$.

A relation $H$ is closed if (the graph of) $H$ is a closed subspace of $\Pi \times \Pi$. For any relation $H$ in $\{\Pi,[\cdot, \cdot]\}$, its adjoint, denoted as $H^{[*]}$, is defined via its graph:

$$
\operatorname{gr} H^{[*]}=\left\{\left\{f, f^{\prime}\right\} \in \Pi \times \Pi:\left[f, g^{\prime}\right]=\left[f^{\prime}, g\right], \quad \forall\left\{g, g^{\prime}\right\} \in \operatorname{gr} H\right\} .
$$

A relation $A$ in $\{\Pi,[\cdot, \cdot]\}$ is symmetric if $A \subseteq A^{[*]}$ and selfadjoint if $A=A^{[*]}$. An operator $V$ from (a Pontryagin space) $\left\{\Pi_{1},[\cdot, \cdot]_{1}\right\}$ to (a Pontryagin space) $\left\{\Pi_{2},[\cdot, \cdot]_{2}\right\}$ is isometric if $[f, g]_{1}=[V f, V g]_{2}$ for all $f, g \in \operatorname{dom} V$. An isometric operator $U$ from $\left\{\Pi_{1},[\cdot, \cdot]_{1}\right\}$ to $\left\{\Pi_{2},[\cdot, \cdot]_{2}\right\}$ is a standard unitary operator if $\operatorname{dom} U=\Pi_{1}$ and $\operatorname{ran} U=\Pi_{2}$.

For a closed relation $H$ in $\{\Pi,[\cdot, \cdot]\}$, the resolvent set, $\rho(H)$, and the spectrum, $\sigma(H)$, are defined as usual:

$\rho(H)=\{z \in \mathbb{C}: \operatorname{ker}(H-z)=\{0\}, \operatorname{ran}(H-z)=\Pi\} \quad$ and $\quad \sigma(H)=\mathbb{C} \backslash \rho(H)$.

Moreover, the point spectrum $\sigma_{p}(H)$ is defined as the set

$$
\sigma_{p}(H)=\{z \in \mathbb{C} \cup\{\infty\}: \exists x(\neq 0) \in \Pi \text { s.t. }\{x, z x\} \in \operatorname{gr}(H)\} .
$$

These sets have the normal properties, see e.g. [4]. Below we also use the convention that $\infty \in \sigma_{p}(H)$ if and only if mul $H \neq\{0\}$ or, equivalently, $0 \in \sigma_{p}\left(H^{-1}\right)$, where $H^{-1}$ stands for the inverse (linear relation) of $H$. Similarly, $\infty \in \rho(H)$ means that $0 \in \rho\left(H^{-1}\right)$ or, equivalently, that $H$ is a bounded everywhere defined operator, i.e., $H \in \mathbf{B}(\Pi)$.

A subspace $\mathfrak{L}$ of $\Pi$ is said to be invariant under a relation $H$ with $\rho(H) \neq \emptyset$, or $H$-invariant for short, if

$$
(H-z)^{-1} \mathfrak{L} \subseteq \mathfrak{L}, \quad \forall z \in \rho(H) .
$$

Here $(H-z)^{-1} \in \mathbf{B}(\Pi)$ is defined via its graph as

$$
\operatorname{gr}\left((H-z)^{-1}\right)=\left\{\left\{f^{\prime}-z f, f\right\} \in \Pi \times \Pi:\left\{f, f^{\prime}\right\} \in \operatorname{gr}(H)\right\} .
$$


Recall that the spectrum and resolvent set $\sigma(A)$ and $\rho(A)$ of a selfadjoint relation $A$ in a Pontryagin space are symmetric with respect to the real line:

$$
\rho(A)=\overline{\rho(A)}, \quad \sigma(A)=\overline{\sigma(A)} \quad \text { and } \quad \sigma_{p}(A)=\overline{\sigma_{p}(A)} .
$$

Moreover, if $\rho(A) \neq \emptyset$ then $\rho(A)$ contains $\mathbb{C} \backslash \mathbb{R}$ except finitely many points; see [4].

Finally $\alpha \in \mathbb{C} \cup\{\infty\}$ is an eigenvalue not of positive type, or ENT for short, of a selfadjoint relation $A$ in a Pontryagin space, if there exists a non-trivial nonpositive $A$-invariant subspace $\mathfrak{L}$ such that $\sigma(A\lceil\mathfrak{L})=\alpha$. Recall that selfadjoint relations in Pontryagin spaces possess at most finitely many ENTs, see e.g. $[9$, Thm. 12.1']. The set of all ENTs of a selfadjoint relation $A$ in $\mathbb{C} \cup\{\infty\}$ is denoted by $\operatorname{ENT}(A)$.

\subsection{Minimal realizations of generalized Nevanlinna functions}

The concept of an operator-valued generalized Nevanlinna function has been introduced and studied by M.G. Krĕ̌n and H. Langer; see [11, 12]. In particular, with some additional analytic assumptions, operator-valued generalized Nevanlinna functions were described as so-called $Q$-functions of symmetric operators in a Pontryagin space. Those additional conditions were removed by allowing selfadjoint relations in model spaces; cf. [3] for the case of matrix functions and [7] for operator-valued functions.

If $A$ is a selfadjoint relation in (a Pontryagin space) $\{\Pi,[\cdot, \cdot]\}$ with a nonempty resolvent set $\rho(A), C$ is a bounded selfadjoint operator in a Hilbert space $\{\mathcal{H},(\cdot, \cdot)\}$ and $\Gamma$ is an everywhere defined operator from $\mathcal{H}$ to $\Pi$, then $F$ defined by

$$
F(z)=C+\overline{z_{0}} \Gamma^{[*]} \Gamma+\left(z-\overline{z_{0}}\right) \Gamma^{[*]}\left(I+\left(z-z_{0}\right)(A-z)^{-1}\right) \Gamma, \quad z, z_{0} \in \rho(A),
$$

is a generalized Nevanlinna function. Conversely, if $F$ is a generalized Nevanlinna function, then there exist $A=A^{[*]}$ with $\rho(A) \neq \emptyset, \Gamma$ and $C$ as above such that (2.2) holds; in this case $C+\overline{z_{0}} \Gamma^{[*]} \Gamma=F\left(z_{0}\right)^{*}=F\left(\overline{z_{0}}\right)$.

If (2.2) holds for some generalized Nevanlinna function $F$, then the pair $\{A, \Gamma\}$ realizes $F$ (at $z_{0}$ ). In particular, in the term realization the realizing space $\{\Pi,[\cdot, \cdot]\}$ is suppressed; also the selection of the arbitrarily fixed point $z_{0}$ is suppressed when it doesn't play a role. With a realizing pair $\{A, \Gamma\}$ (at $z_{0}$ ) we associate a bounded operator-valued function $\Gamma_{z}$, called the $\gamma$-field associated with $\{A, \Gamma\}$, via

$$
\Gamma_{z}:=\left(I+\left(z-z_{0}\right)(A-z)^{-1}\right) \Gamma, \quad z \in \rho(A) .
$$

Using the $\gamma$-field and the resolvent identity, (2.2) can be rewritten into a symmetric form:

$$
\frac{F(z)-F(w)^{*}}{z-\bar{w}}=\Gamma_{w}^{[*]} \Gamma_{z}, \quad z, w \in \rho(A)
$$


The pair $\{A, \Gamma\}$ is said to realize $F$ (as in (2.2)) minimally if

$$
\Pi=\text { c.l.s. }\left\{\Gamma_{z} h: z \in \rho(A), \quad h \in \mathcal{H}\right\} .
$$

For the existence of a minimal realization for any generalized Nevanlinna function see e.g. [7, Thm. 4.2].

By means of a minimal realization the index of a generalized Nevanlinna function can be characterized: $F$ is a generalized Nevanlinna function with index $\kappa, F \in \mathfrak{N}_{\kappa}(\mathcal{H})$, if the negative index of the realizing (Pontryagin) space for any minimal realization is $\kappa$. In fact, all minimal realizations are connected by means of (standard) unitary operators.

Proposition 2.1. ([7, Thm. 3.2]) Let $\left\{A_{i}, \Gamma_{i}\right\}$ realize $F \in \mathfrak{N}_{\kappa}(\mathcal{H})$ minimally for $i=$ 1,2 . Then there exists a standard unitary operator from $\left\{\Pi_{1},[\cdot, \cdot]_{1}\right\}$ to $\left\{\Pi_{2},[\cdot, \cdot]_{2}\right\}$ such that $A_{2}=U A_{1} U^{-1}$ and $\Gamma_{2}=U \Gamma_{1}$.

For a generalized Nevanlinna function $F$ the notation $\rho(F)$ and $\sigma(F)$ is used to denote the domain of holomorphy of $F$ in $\mathbb{C} \cup\{\infty\}$ and its complement (in $\mathbb{C} \cup\{\infty\}$ ), respectively. In particular, (2.2) implies that

$$
\rho(A) \subseteq \rho(F) \text { and } \sigma(F) \subseteq \sigma(A) .
$$

For minimal realizations the reverse inclusions also hold.

Theorem 2.2. ([11, Satz 4.4]) Let $F \in \mathfrak{N}_{\kappa}(\mathcal{H})$ be minimally realized by $\{A, \Gamma\}$. Then $\rho(A)=\rho(F)$.

Finally, $\alpha \in \mathbb{C} \cup\{\infty\}$ is a generalized pole of a generalized Nevanlinna function $F$ if $\alpha \in \sigma_{p}(A)$ for any minimal realization $\{A, \Gamma\}$ of $F$. Furthermore, the set of generalized poles of not of positive type of $F, \operatorname{GPNT}(F)$, is defined to be $\operatorname{ENT}(A)$ (see Section 2.1). Note that Proposition 2.1 guarantees that these concepts are well-defined.

\subsection{Reduction of non-minimal realizations}

Realizations for a generalized Nevanlinna function need not be minimal. For instance, if the negative index of the realizing Pontryagin space is greater than the negative index of a generalized Nevanlinna function, then the realization is not minimal. Even if the negative index of the realizing space is equal to the negative index of a generalized Nevanlinna function, the realization might still be non-minimal; cf. Section 2.4 below. The following operator-valued analog of [16, Prop. 2.2] shows how non-minimal realizations can be reduced to minimal ones; see also [11] and [7, Section 2].

Proposition 2.3. Let $\{A, \Gamma\}$ realize $F \in \mathfrak{N}_{\kappa}(\mathcal{H})$ and let $\kappa_{m}$ denote the negative index of the realizing Pontryagin space $\{\Pi,[\cdot, \cdot]\}$. Moreover, with

$$
\mathfrak{M}:=\operatorname{span}\left\{\left(I+\left(z-z_{0}\right)(A-z)^{-1}\right) \Gamma h: z \in \rho(A), \quad h \in \mathcal{H}\right\},
$$


define $\mathfrak{L}, \Pi_{s}$ and $\Pi_{r}$ as

$$
\mathfrak{L}=(\operatorname{clos} \mathfrak{M}) \cap \mathfrak{M}^{[\perp]}, \quad \Pi_{s}=(\operatorname{clos} \mathfrak{M}) / \mathfrak{L} \quad \text { and } \quad \Pi_{r}=\mathfrak{M}^{[\perp]} / \mathfrak{L} .
$$

Then the following statements hold:

(i) $\mathfrak{L}$ is an A-invariant neutral subspace of $\{\Pi,[\cdot, \cdot]\}$ with $\kappa_{\mathfrak{L}}:=\operatorname{dim} \mathfrak{L} \leq \kappa_{m}$;

(ii) $A_{s}$ and $A_{r}$, defined via

$$
\begin{aligned}
& \operatorname{gr} A_{s}=\left\{\left\{f+[\mathfrak{L}], f^{\prime}+[\mathfrak{L}]\right\}:\left\{f, f^{\prime}\right\} \in \operatorname{gr} A \cap\left(\Pi_{s} \times \Pi_{s}\right)\right\} ; \\
& \operatorname{gr} A_{r}=\left\{\left\{f+[\mathfrak{L}], f^{\prime}+[\mathfrak{L}]\right\}:\left\{f, f^{\prime}\right\} \in \operatorname{gr} A \cap\left(\Pi_{r} \times \Pi_{r}\right)\right\},
\end{aligned}
$$

are selfadjoint relations in the Pontryagin spaces $\left\{\Pi_{s},[\cdot, \cdot]\right\}$ and $\left\{\Pi_{r},[\cdot, \cdot]\right\}$ with negative index $\kappa$ and $\kappa_{m}-\kappa-\kappa_{\mathfrak{L}}$, respectively;

(iii) $\left\{A_{s}, \Gamma+[\mathfrak{L}]\right\}$ realizes $f$ minimally;

(iv) $\mathfrak{M}^{[\perp]}$ is the largest $A$-invariant subspace contained in $\operatorname{ker} \Gamma^{[*]}$.

Proof. (i) Let $\mathfrak{M}$ be as in the statement, then $(A-\xi)^{-1} \mathfrak{M} \subseteq \mathfrak{M}$ for every $\xi \in \rho(A)$ by the resolvent identity. From the preceding inclusion it follows by elementary arguments that $\left((A-\xi)^{-1}\right)^{[*]} \mathfrak{M}^{[\perp]} \subseteq \mathfrak{M}^{[\perp]}$ or, equivalently, using the selfadjointness of $A$ that $(A-\bar{\xi})^{-1} \mathfrak{M}^{[\perp]} \subseteq \mathfrak{M}^{[\perp]}$. Another application of the same argument yields that $(A-\xi)^{-1} \operatorname{clos} \mathfrak{M} \subseteq \operatorname{clos} \mathfrak{M}$. Since $\rho(A)$ is symmetric with respect to the real line for selfadjoint relations, see $(2.1), \mathfrak{M}$, clos $\mathfrak{M}$ and $\mathfrak{M}^{[\perp]}$ are $A$-invariant and, hence, $\mathfrak{L}$ is $A$-invariant, too.

(ii) Since $\mathfrak{L}$ is neutral in a Pontryagin space, it is a finite-dimensional (closed) subspace. Therefore $\left\{\mathfrak{L}^{[\perp]} / \mathfrak{L},[\cdot, \cdot]\right\}$ is a Pontryagin space with negative index $\kappa_{m}-$ $\kappa_{\mathfrak{L}}$, see [1, Ch. 1: Cor. 9.14]. A calculation, using the $A$-invariance and neutrality of $\mathfrak{L}$, shows that $A_{\mathfrak{L}}$, defined via

$$
\operatorname{gr}\left(A_{\mathfrak{L}}\right)=\left\{\left\{f+[\mathfrak{L}], f^{\prime}+[\mathfrak{L}]\right\} \in \mathfrak{L}^{[\perp]} / \mathfrak{L} \times \mathfrak{L}^{[\perp]} / \mathfrak{L}:\left\{f, f^{\prime}\right\} \in \operatorname{gr} A \cap\left(\mathfrak{L}^{[\perp]} \times \mathfrak{L}^{[\perp]}\right)\right\}
$$

is a symmetric linear relation in the introduced quotient space. To establish that $A$ is selfadjoint, it suffices by $[4$, Thm. 4.6] to show that

$$
\rho(A) \subseteq \rho\left(A_{\mathfrak{L}}\right) .
$$

Let $z \in \rho(A)$ be arbitrary. Since $\mathfrak{L}$ is $A$-invariant (see (i)), $\mathfrak{L}^{[\perp]}$ is also $A$-invariant and $\mathfrak{L}^{[\perp]} \subseteq \operatorname{ran}(A-z)$, because $z \in \rho(A)$ by assumption. Thus for every $g \in \mathfrak{L}^{[\perp]}$ there exists $\left\{f, f^{\prime}\right\} \in A$, such that $g=f^{\prime}-z f$. Now the $A$-invariance of $\mathfrak{L}^{[\perp]}$ implies that $f=(A-z)^{-1} g \in \mathfrak{L}^{[\perp]}$ and thus also $f^{\prime} \in \mathfrak{L}^{[\perp]}$. Therefore,

$$
\mathfrak{L}^{[\perp]} \subseteq\left\{f^{\prime}-z f:\left\{f, f^{\prime}\right\} \in \operatorname{gr} A \cap\left(\mathfrak{L}^{[\perp]} \times \mathfrak{L}^{[\perp]}\right)\right\} .
$$

Consequently, $\operatorname{ran}\left(A_{\mathfrak{L}}-z\right)=\mathfrak{L}^{[\perp]} / \mathfrak{L}$ and this implies that $z \in \rho\left(A_{\mathfrak{L}}\right)$. Since $z \in \rho(A)$ was arbitrary, the above argument shows that (2.6) holds.

Now $\Gamma_{\mathfrak{L}}$, defined via $\Gamma_{\mathfrak{L}} h:=\Gamma h+[\mathfrak{L}]$ for $h \in \mathcal{H}$, is an everywhere defined mapping from $\mathcal{H}$ to $\mathfrak{L}^{[\perp]} / \mathfrak{L}$. Using $\Gamma_{\mathfrak{L}}$ and $A_{\mathfrak{L}}$ define the subspace $\mathfrak{M}_{\mathfrak{L}}$ of $\mathfrak{L}^{[\perp]} / \mathfrak{L}$ 
as

$$
\mathfrak{M}_{\mathfrak{L}}:=\operatorname{span}\left\{\left(I+\left(z-z_{0}\right)\left(A_{\mathfrak{L}}-z\right)^{-1}\right) \Gamma_{\mathfrak{L}} h: z \in \rho\left(A_{\mathfrak{L}}\right), \quad h \in \mathcal{H}\right\} .
$$

By means of $\mathfrak{M}_{\mathfrak{L}}$ introduce in $\left\{\mathfrak{L}^{[\perp]} / \mathfrak{L},[\cdot, \cdot]\right\}$ the subspaces $\Pi_{s}:=\operatorname{clos} \mathfrak{M}_{\mathfrak{L}}$ and $\Pi_{r}:=\Pi_{s}^{[\perp]_{\mathfrak{L}}}$; here $[\perp]_{\mathfrak{L}}$ denotes the orthogonal complement in $\left\{\mathfrak{L}^{[\perp]} / \mathfrak{L},[\cdot, \cdot]\right\}$. Then clearly $\Pi_{s}=\operatorname{clos}(\mathfrak{M}) / \mathfrak{L}$ and $\Pi_{r}=\mathfrak{M}^{[\perp]} / \mathfrak{L}$. Since $\mathfrak{L}=\operatorname{clos}(\mathfrak{M}) \cap \mathfrak{M}^{[\perp]}, \Pi_{s}$ and $\Pi_{r}$ are non-degenerate. Therefore $\left\{\Pi_{s},[\cdot, \cdot]\right\}$ and $\left\{\Pi_{r},[\cdot, \cdot]\right\}$ are Pontryagin spaces, see [1, Ch. 1: Thm. $7.16 \&$ Thm. 9.9]. The same arguments used in (i) yield

$$
\left(A_{\mathfrak{L}}-\xi\right)^{-1} \Pi_{s} \subseteq \Pi_{s} \quad \text { and } \quad\left(A_{\mathfrak{L}}-\xi\right)^{-1} \Pi_{r} \subseteq \Pi_{r}, \quad \xi \in \rho\left(A_{\mathfrak{L}}\right) \supseteq \rho(A) .
$$

Let $A_{s}$ and $A_{r}$ be as in (ii) with $\Pi_{s}$ and $\Pi_{r}$ as defined following (2.7), then $A_{s}$ and $A_{r}$, being restrictions of the selfadjoint relation $A_{\mathfrak{L}}$, are symmetric. Moreover, (2.8) together with the decomposition $\mathfrak{L}^{[\perp]} / \mathfrak{L}=\Pi_{s}[\dot{+}] \Pi_{r}$ implies that $\rho\left(A_{s}\right) \cap \mathbb{C}_{+}$, $\rho\left(A_{s}\right) \cap \mathbb{C}_{-}, \rho\left(A_{r}\right) \cap \mathbb{C}_{+}$and $\rho\left(A_{r}\right) \cap \mathbb{C}_{-}$are all non-empty. Therefore $A_{s}$ and $A_{r}$ are selfadjoint relations; again cf. [4]. The last assertion on the negative indices of the Pontryagin spaces is a consequence of the result in (iii) combined with the fact that the negative index of the Pontryagin space $\left\{\mathfrak{L}^{[\perp]} / \mathfrak{L},[\cdot, \cdot]\right\}$ is $\kappa_{m}-\operatorname{dim} \mathfrak{L}=\kappa_{m}-\kappa_{\mathfrak{L}}$.

(iii) Let $\Gamma_{z}$ be the $\gamma$-field associated with the realization $\{A, \Gamma\}$ as in (2.3). Then for every $\omega_{g}, \omega_{h} \in \mathfrak{L}$ we have by definition of $\mathfrak{L}$ that

$$
\left[\Gamma_{z} h+\omega_{h}, \Gamma_{w} g+\omega_{g}\right]=\left[\Gamma_{z} h, \Gamma_{w} g\right]=g^{*} \frac{F(z)-F(w)^{*}}{z-\bar{w}} h, \quad g, h \in \mathcal{H} .
$$

Hence, $\left\{A_{s}, \Gamma_{\mathfrak{L}}\right\}$ realizes $F$, see $(2.4)$. Moreover, this realization is minimal by construction, see the proof of (ii). Therefore the negative index of $\left\{\Pi_{s},[\cdot, \cdot]\right\}$ is $\kappa$ by Proposition 2.1 and the discussion preceding it.

(iv) In (i) it has been established that $\mathfrak{M}^{[\perp]}$ is $A$-invariant. The inclusion $\mathfrak{M}^{[\perp]} \subseteq \operatorname{ker} \Gamma^{[*]}$ follows directly from the fact that $\operatorname{ran} \Gamma \subseteq \mathfrak{M}$. Therefore to prove the assertion it suffices to show that all $A$-invariant subspaces $\mathfrak{N}$ contained in $\operatorname{ker} \Gamma^{[*]}$ are orthogonal to $\mathfrak{M}$. Let $\mathfrak{N}$ be any such subspace. Then for all $h \in \mathcal{H}$ and $z \in \rho(A)$

$$
\left[\left(I+\left(z-z_{0}\right)(A-z)^{-1}\right) \Gamma h, \mathfrak{N}\right]=\left(h, \Gamma^{[*]}\left(I+\left(\bar{z}-\overline{z_{0}}\right)(A-\bar{z})^{-1}\right) \mathfrak{N}\right)=0 .
$$

This shows that $\mathfrak{N} \subseteq \mathfrak{M}^{[\perp]}$.

Corollary 2.4. Let $F \in \mathfrak{N}_{\kappa}(\mathcal{H})$ be realized by $\{A, \Gamma\}$ and let $\kappa_{m}$ denote the negative index of the realizing Pontryagin space $\{\Pi,[\cdot, \cdot]\}$. Then

$$
\kappa_{m}-\kappa=\max _{\mathfrak{N}}\left\{\operatorname{dim} \mathfrak{N}: \mathfrak{N} \text { is A-invariant, } \mathfrak{N} \subseteq \operatorname{ker} \Gamma^{[*]}\right\} ;
$$

here the maximum is over all nonpositive subspaces $\mathfrak{N}$ of $\{\Pi,[\cdot, \cdot]\}$.

Proof. Using the notation as in Proposition 2.3, Proposition 2.3 (ii) shows that $\kappa_{m}-\kappa=\operatorname{dim} \mathfrak{L}+\kappa_{r}$; here $\kappa_{r}$ is defined to be the negative index of the Pontryagin space $\left\{\Pi_{r},[\cdot, \cdot]\right\}$. Since the negative index of the subspace $\mathfrak{M}^{[\perp]}$ of $\{\Pi,[\cdot, \cdot]\}$ is equal 
to $\operatorname{dim} \mathfrak{L}+\kappa_{r}$ and $\left\{\mathfrak{N}: \mathfrak{N}\right.$ is $A$-invariant, $\left.\mathfrak{N} \subseteq \operatorname{ker} \Gamma^{[*]}\right\} \subseteq \mathfrak{M}^{[\perp]}$ by Proposition 2.3 (iv), the statement is proven if the existence of a nonpositive $A$-invariant subspace of dimension $\operatorname{dim} \mathfrak{L}+\kappa_{r}$ contained in $\operatorname{ker} \Gamma^{[*]}$ is established.

Since $A_{r}$, the restriction of $A$ to $\left\{\Pi_{r},[\cdot, \cdot]\right\}$ (see Proposition 2.3 (ii)), is a selfadjoint relation in the Pontryagin space $\left\{\Pi_{r},[\cdot, \cdot]\right\}$, the invariant subspace theorem states that there exists a $\kappa_{r}$-dimensional nonpositive subspace $\mathfrak{L}_{r}$ of $\left\{\Pi_{r},[\cdot, \cdot]\right\}$ which is $A_{r}$-invariant, see e.g. [9, Thm. 12.1']. Therefore $\mathfrak{N}:=\mathfrak{L}_{r}+\mathfrak{L}$ is a $\left(\operatorname{dim} \mathfrak{L}+\kappa_{r}\right)$-dimensional nonpositive $A$-invariant subspace contained in $\mathfrak{M}^{[\perp]}$.

\subsection{The sum of generalized Nevanlinna functions}

A particular situation where non-minimal realizations may be encountered is when the sum of generalized Nevanlinna functions is considered; cf. [6]. Let $F_{i} \in \mathfrak{N}_{\kappa_{i}}(\mathcal{H})$ be (minimally) realized by $\left\{A_{i}, \Gamma_{i}\right\}$, for $i=1,2$. Then the sum $F_{1}+F_{2}$ is realized by $\left\{A_{1} \widehat{\oplus} A_{2}, \operatorname{col}\left(\Gamma_{1}, \Gamma_{2}\right)\right\}$, where

$$
\begin{aligned}
\operatorname{gr}\left(A_{1} \widehat{\oplus} A_{2}\right) & =\left\{\left\{\left\{f_{1}, f_{2}\right\},\left\{f_{1}^{\prime}, f_{2}^{\prime}\right\}\right\}:\left\{f_{i}, f_{i}^{\prime}\right\} \in \operatorname{gr}\left(A_{i}\right)\right\} ; \\
\operatorname{col}\left(\Gamma_{1}, \Gamma_{2}\right) h & =\left(\begin{array}{l}
\Gamma_{1} h \\
\Gamma_{2} h
\end{array}\right) .
\end{aligned}
$$

Here the realizing space is $\left\{\Pi_{\text {sum }},[\cdot, \cdot]_{\text {sum }}\right\}$ where $\Pi_{\text {sum }}=\Pi_{1} \times \Pi_{2}$ and

$$
\left[\left\{f_{1}, f_{2}\right\},\left\{g_{1}, g_{2}\right\}\right]_{\text {sum }}=\left[f_{1}, g_{1}\right]_{1}+\left[f_{2}, g_{2}\right]_{2}, \quad\left\{f_{1}, f_{2}\right\},\left\{g_{1}, g_{2}\right\} \in \Pi_{1} \times \Pi_{2} . \quad \text { (2.10) }
$$

To see this note that

$$
\begin{aligned}
& \left(\operatorname{col}\left(\Gamma_{1}, \Gamma_{2}\right)\right)^{[*]}\left(I+\left(z-z_{0}\right)\left(A_{1} \widehat{\oplus} A_{2}-z\right)^{-1}\right) \operatorname{col}\left(\Gamma_{1}, \Gamma_{2}\right) \\
= & \left(\begin{array}{c}
\Gamma_{1} \\
\Gamma_{2}
\end{array}\right)^{[*]}\left(\begin{array}{cc}
I+\left(z-z_{0}\right)\left(A_{1}-z\right)^{-1} & 0 \\
0 & I+\left(z-z_{0}\right)\left(A_{2}-z\right)^{-1}
\end{array}\right)\left(\begin{array}{c}
\Gamma_{1} \\
\Gamma_{2}
\end{array}\right) \\
= & \Gamma_{1}^{[*]}\left(I+\left(z-z_{0}\right)\left(A_{1}-z\right)^{-1}\right) \Gamma_{1}+\Gamma_{2}^{[*]}\left(I+\left(z-z_{0}\right)\left(A_{2}-z\right)^{-1}\right) \Gamma_{2} \\
= & \frac{F_{1}(z)-F_{1}\left(\overline{z_{0}}\right)}{z-\overline{z_{0}}}+\frac{F_{2}(z)-F_{2}\left(\overline{z_{0}}\right)}{z-\overline{z_{0}}}=\frac{F_{1}(z)+F_{2}(z)-\left(F_{1}\left(\overline{z_{0}}\right)+F_{2}\left(\overline{z_{0}}\right)\right)}{z-\overline{z_{0}}},
\end{aligned}
$$

where in the third step (2.2) was used. In view of (2.2) this calculation shows that $\left\{A_{1} \widehat{\oplus} A_{2}, \operatorname{col}\left(\Gamma_{1}, \Gamma_{2}\right)\right\}$ realizes $F_{1}+F_{2}$; cf. [6, Prop. 4.1]. In particular, $F_{1}+F_{2} \in$ $\mathfrak{N}_{\kappa_{\text {sum }}}(\mathcal{H})$ where $\kappa_{\text {sum }} \leq \kappa_{1}+\kappa_{2} ;$ cf. Proposition 2.3.

Notice, conversely, that if $\{A, \Gamma\}$ realizes the function $F \in \mathfrak{N}_{\kappa}(\mathcal{H})$ and there exists a decomposing (regular) subspace $\Pi_{1}$ of $\Pi$, i.e. $\Pi=\Pi_{1}[\dot{+}] \Pi_{2}$ with $\Pi_{2}=\Pi_{1}^{[\perp]}$, which also reduces $A, A=A_{1} \widehat{\oplus} A_{2}$, then $\left\{A_{1}, P_{1} \Gamma\right\}$ and $\left\{A_{2}, P_{2} \Gamma\right\}$, where $P_{j}$ with $j=1,2$ is the $\Pi$-orthogonal projection onto $\Pi_{j}$, produce realizations for generalized Nevanlinna functions $F_{1}$ and $F_{2}$ such that $F=F_{1}+F_{2}$.

Proposition 2.5 below contains sufficient conditions for the index of $F_{1}+F_{2}$ to be the sum of the indices of $F_{1}$ and $F_{2}$; see [2, Prop. 3.2] for a similar statement for matrix-valued generalized Nevanlinna functions. 
Proposition 2.5. Let $F_{1} \in \mathfrak{N}_{\kappa_{1}}(\mathcal{H}), F_{2} \in \mathfrak{N}_{\kappa_{2}}(\mathcal{H})$ and assume that $F_{1}$ and $F_{2}$ do not have a generalized pole in common. Then $F_{1}+F_{2} \in \mathfrak{N}_{\kappa_{1}+\kappa_{2}}(\mathcal{H})$.

Proof. Let $\left\{A_{i}, \Gamma_{i}\right\}$ be a minimal realization for $F_{i}$ where the realizing space is $\left\{\Pi_{i},[\cdot, \cdot]_{i}\right\}$, for $i=1,2$. Then, as the discussion preceding this statement demonstrated, $F_{1}+F_{2}$ is realized by $\{A, \Gamma\}:=\left\{A_{1} \widehat{\oplus} A_{2}, \operatorname{col}\left(\Gamma_{1}, \Gamma_{2}\right)\right\}$ where the realizing space is the Pontryagin space $\{\Pi,[\cdot, \cdot]\}:=\left\{\Pi_{\text {sum }},[\cdot, \cdot]_{\text {sum }}\right\}$ whose negative index is $\kappa_{1}+\kappa_{2}$, see (2.10). Hence $F_{1}+F_{2}$ is a generalized Nevanlinna function. In order to establish that its index is $\kappa_{1}+\kappa_{2}$, the non-minimal part of its realization $\{A, \Gamma\}$ should be investigated; cf. Proposition 3.1. But first note that if $P_{1}$ and $P_{2}$ are the orthogonal projections onto $\Pi_{1}$ and $\Pi_{2}$ in $\Pi_{\text {sum }}$, then

$$
\left(A_{1} \widehat{\oplus} A_{2}-z\right)^{-1} P_{i}=\left(A_{i}-z\right)^{-1} P_{i}=P_{i}\left(A_{1} \widehat{\oplus} A_{2}-z\right)^{-1}, \quad i=1,2,
$$

see $(2.9)$. Denote by $\mathfrak{M}^{[\perp]}$ the non-minimal part of the realization $\{A, \Gamma\}$ as in Proposition 2.3. If $\mathfrak{L}:=\operatorname{clos}(\mathfrak{M}) \cap \mathfrak{M}^{[\perp]} \neq\{0\}$, then $\mathfrak{L}$, being finite-dimensional and $A$-invariant (see Proposition 2.3 (i)), contains an eigenvector $x$ for $A=A_{1} \widehat{\oplus} A_{2}$ such that $x \in \operatorname{ker} \Gamma^{[*]}$. But, then (2.11) implies that $P_{1} x$ and $P_{2} x$ are eigenvectors for $A_{1}$ and $A_{2}$, respectively. Since $\sigma_{p}\left(A_{1}\right) \cap \sigma_{p}\left(A_{2}\right)=\emptyset$ by assumption, this implies that either of the two vectors is zero; say $P_{2} x=0$. Thus $P_{1} x$ is an eigenvector for $A_{1}, P_{2} x=0$ and $x \in \operatorname{ker} \Gamma^{[*]}$. The last two conditions together yield that $P_{1} x \in \operatorname{ker} \Gamma_{1}^{[*]}$; cf. (2.9). But then the realization $\left\{A_{1}, \Gamma_{1}\right\}$ for $F_{1}$ is not minimal by Proposition 2.3; in contradiction to the assumption. I.e., $\mathfrak{L}=\{0\}$.

Therefore $\mathfrak{M}^{[\perp]}$ is $A$-invariant and $\left\{\mathfrak{M}^{[\perp]},[\cdot, \cdot]\right\}$ is a Pontryagin space, see Proposition 2.3 (ii). The exact same argument as used in the preceding paragraph shows that $\sigma_{p}\left(A\left\lceil_{\mathfrak{M}[\perp]}\right)=\emptyset\right.$. Hence Pontryagin's invariant subspace theorem (applied to the selfadjoint relation $A \uparrow_{\mathfrak{M}[\perp]}$ in $\left.\left\{\mathfrak{M}^{[\perp]},[\cdot, \cdot]\right\}\right)$ implies that $\left\{\mathfrak{M}^{[\perp]},[\cdot, \cdot]\right\}$ is a Hilbert space, see e.g. [9, Thm. 12.1']. Consequently, the statement holds by Proposition 2.3 (ii); cf. Corollary 2.4 .

Extending upon Proposition 2.5, the following result shows when a minimal realization for $F_{1}+F_{2}$ can be obtained when starting from minimal realizations for $F_{1}$ and $F_{2}$.

Proposition 2.6. Let $\left\{A_{i}, \Gamma_{i}\right\}$ minimally realize the generalized Nevanlinna function $F_{i} \in \mathfrak{N}_{\kappa_{i}}(\mathcal{H})$, for $i=1,2$, and assume that

$$
\sigma_{p}\left(A_{1}\right) \cap \sigma_{p}\left(A_{2}\right)=\emptyset \quad \text { and } \quad \sigma\left(A_{1}\right) \cap \sigma\left(A_{2}\right)=\left\{\gamma_{1}, \ldots, \gamma_{n}\right\} \subseteq \mathbb{R} \cup\{\infty\} .
$$

Then $F_{1}+F_{2} \in \mathfrak{N}_{\kappa_{1}+\kappa_{2}}(\mathcal{H})$ is minimally realized by $\left\{A_{1} \widehat{\oplus} A_{2}, \operatorname{col}\left(\Gamma_{1}, \Gamma_{2}\right)\right\}$.

Proof. As the above discussion demonstrated, $F_{1}+F_{2}$ is realized by $\{A, \Gamma\}:=$ $\left\{A_{1} \widehat{\oplus} A_{2}, \operatorname{col}\left(\Gamma_{1}, \Gamma_{2}\right)\right\}$ where the realizing space is the Pontryagin space $\{\Pi,[\cdot, \cdot]\}:=$ $\left\{\Pi_{\text {sum }},[\cdot, \cdot]_{\text {sum }}\right\}$ whose negative index is $\kappa_{1}+\kappa_{2}$, see $(2.10)$. To prove the minimality of the realization for $F_{1}+F_{2}$ let $\mathfrak{M}$ be as in Proposition 2.3. 
Since the index of $F_{1}+F_{2}$ is equal to the negative index of $\{\Pi,[\cdot, \cdot]\}$ by Proposition 2.5, Proposition 2.3 yields that $\left\{\mathfrak{M}^{[\perp]},[\cdot, \cdot]\right\}$ is a Hilbert space and that $A_{r}$, defined via $\operatorname{gr}\left(A_{r}\right)=\operatorname{gr}(A) \cap\left(\mathfrak{M}^{[\perp]} \times \mathfrak{M}^{[\perp]}\right)$, is a selfadjoint relation in $\left\{\mathfrak{M}^{[\perp]},[\cdot, \cdot]\right\}$. In particular, $\sigma\left(A_{r}\right) \subseteq \mathbb{R} \cup\{\infty\}$. We claim that

$$
\sigma\left(A_{r}\right) \subseteq \sigma\left(A_{1}\right) \text { and } \sigma\left(A_{r}\right) \subseteq \sigma\left(A_{2}\right) .
$$

If the first inclusion does not hold, then, since $\sigma\left(A_{1}\right) \cap(\mathbb{R} \cup\{\infty\})$ and $\sigma\left(A_{r}\right)$ are closed subsets of $\mathbb{R} \cup\{\infty\}$, there exists a closed interval $\Delta=[a, b]$ of $\mathbb{R}$ such that

$$
\Delta \cap \sigma\left(A_{r}\right) \neq \emptyset \text { and } \Delta \subseteq \rho\left(A_{1}\right) .
$$

Let $E_{t}$ be the spectral family of $A_{r}$ and let $P_{i}$ be the orthogonal projections onto $\Pi_{i}$ in $\Pi$, for $i=1,2$. Then the assumption $\Delta \cap \sigma\left(A_{r}\right) \neq \emptyset$ implies that

$$
\mathfrak{L}:=\left(E_{b}-E_{a}\right) \mathfrak{M}^{[\perp]} \neq\{0\} .
$$

Consider $\mathfrak{L}_{1}:=P_{1} \mathfrak{L} \subseteq \Pi_{1}$. Then, on the one hand,

$$
\sigma\left(A\left\lceil_{\mathfrak{L}_{1}}\right) \subseteq \sigma\left(A \Upsilon_{\mathfrak{L}}\right) \subseteq \Delta \subseteq \rho\left(A_{1}\right) .\right.
$$

On the other hand, the $A_{1}$-invariance of $\mathfrak{L}_{1}$ implies that $\sigma\left(A\left\lceil\mathfrak{L}_{1}\right) \subseteq \sigma\left(A_{1}\right)\right.$. The preceding two results together imply that $\mathfrak{L}_{1}=\{0\}$; cf. (2.13). In other words, $\mathfrak{L} \subseteq\{0\} \times \Pi_{2}$. But then $\mathfrak{L} \subseteq \operatorname{ker} \Gamma_{2}^{[*]}$, because $\mathfrak{L} \subseteq \mathfrak{M}^{[\perp]} \subseteq \operatorname{ker} \Gamma^{[*]}$. Consequently, the realization $\left\{A_{2}, \Gamma_{2}\right\}$ is not minimal. This contradiction shows that the first inclusion in (2.12) holds. By symmetry the second inclusion also holds.

Combining the inclusions from (2.12) together with the assumption about $\sigma\left(A_{1}\right) \cap \sigma\left(A_{1}\right)$ yields that $\sigma\left(A_{r}\right)$ consists at most of isolated points. I.e., all the spectrum of $A_{r}$ is point spectrum. Let $x$ be an eigenvector for $A_{r}$. Then $P_{1} x$ and $P_{2} x$ are eigenvectors for $A_{1}$ and $A_{2}$, respectively. Since $\sigma_{p}\left(A_{1}\right) \cap \sigma_{p}\left(A_{2}\right)=\emptyset$, either $P_{1} x$ or $P_{2} x$ should be equal to zero. Assume the latter. Since $x \in \mathfrak{M}^{[\perp]} \subseteq \operatorname{ker} \Gamma^{[*]}$ (see Proposition 2.3), it follows that $x=P_{1} x \subseteq \operatorname{ker} \Gamma_{1}^{[*]}$; but this is in contradiction to the assumed minimality of the realization $\left\{A_{1}, \Gamma_{1}\right\}$ of $F_{1}$.

\section{Decompositions of generalized Nevanlinna functions}

For $\alpha, \beta \in \mathbb{C} \cup\{\infty\}$, with $\alpha \neq \beta$, and for non-orthogonal vectors $\eta$ and $\xi$ in a Hilbert space $\mathcal{H}$ define the operator-valued rational function $R$ as:

$$
R(z ; \alpha, \beta, \eta, \xi)=I-P+\frac{z-\alpha}{z-\beta} P, \quad P=\frac{\xi \eta^{*}}{\eta^{*} \xi}, \quad \eta^{*} \xi \neq 0 ;
$$

here $R(z ; \infty, \beta, \eta, \xi)$ and $R(z ; \alpha, \infty, \eta, \xi)$ should be interpreted to be $I-P+(z-$ $\beta)^{-1} P$ and $I-P+(z-\alpha) P$, respectively. Note that

$$
(R(z ; \alpha, \beta, \eta, \xi))^{\#}=R(z ; \bar{\alpha}, \bar{\beta}, \xi, \eta) \quad \text { and } \quad(R(z ; \alpha, \beta, \eta, \xi))^{-1}=(R(z ; \beta, \alpha, \eta, \xi)) ;
$$

here for any operator-valued function $Q(z), Q^{\#}(z)$ is defined to be $Q(\bar{z})^{*}$. 
With this notation, (realizations for) products of the form $R^{\#} F R$, where $R(z)=R(z ; \alpha, \beta, \eta, \xi)$, are investigated in the first subsection. In the second subsection these considerations are combined with a factorization from [14] to decompose generalized Nevanlinna functions with respect to their analytic behavior as stated in Theorem 1.1. These results are in turn used to prove Theorem 1.2 in the third and final subsection.

\subsection{Multiplication with an order one term}

Here an explicit realization for $R^{\#} F R$, where $R$ is as in (3.1), is generated from any given realization for $F \in \mathfrak{N}_{\kappa}(\mathcal{H})$. This realization expresses can be seen as a modification and extension of [16, Thm. 1.3] from scalar-valued to operator-valued functions. Note that the explicit resolvent formula in Proposition 3.1 reflects how the invariant subspaces of the realizing relation for $R^{\#} F R$ are connected to the invariant subspaces of the realizing relation for the original function $F$.

Proposition 3.1. Let $F \in \mathfrak{N}_{\kappa}(\mathcal{H})$ be realized by $\{A, \Gamma\}$ at $z_{0} \in \rho(A) \backslash\{\beta, \bar{\beta}\}$, where $\alpha, \beta \in \mathbb{C} \cup\{\infty\}$ satisfy $\alpha \neq \bar{\beta}$, and let $\xi, \eta \in \mathcal{H}$ satisfy $\eta^{*} \xi \neq 0$. Then $F_{R}:=R^{\#} F R$, where $R(z)=R(z ; \bar{\alpha}, \beta, \eta, \xi)$ as in $(3.1)$, is realized by $\left\{A_{R}, \Gamma_{R}\right\}$ which are defined for $z \in \rho(A) \backslash\{\beta, \bar{\beta}\}$ via

$$
\left(A_{R}-z\right)^{-1}=\left(\begin{array}{ccc}
\frac{1}{\bar{\beta}-z} & \frac{\xi^{*} \Gamma_{\bar{z}}^{[*]}}{\bar{\beta}-z} & \frac{\xi^{*} F(z) \xi}{(\bar{\beta}-z)(\beta-z)} \\
0 & (A-z)^{-1} & \frac{\Gamma_{z} \xi}{\beta-z} \\
0 & 0 & \frac{1}{\beta-z}
\end{array}\right), \quad \Gamma_{R}=\left(\begin{array}{c}
\frac{\xi^{*} F\left(z_{0}\right)}{\bar{\beta}-z_{0}} R\left(z_{0}\right) \\
\Gamma R\left(z_{0}\right) \\
\bar{\alpha}-\beta \\
\bar{\beta}-z_{0} \\
\frac{\eta^{*}}{\eta^{*} \xi}
\end{array}\right) .
$$

Here the realizing space $\left\{\Pi_{2},[\cdot, \cdot]_{2}\right\}$ of $\left\{A_{R}, \Gamma_{R}\right\}$ is defined as $[g, h]_{2}:=\left[g_{c}, h_{c}\right]+g_{r} \overline{h_{l}}+g_{l} \overline{h_{r}}, \quad g=\left\{g_{l}, g_{c}, g_{r}\right\}, h=\left\{h_{l}, h_{c}, h_{r}\right\} \in \Pi_{2}:=\mathbb{C} \times \Pi \times \mathbb{C}$, where $\{\Pi,[\cdot, \cdot]\}$ is the realizing space of $\{A, \Gamma\}$.

Recall that $\Gamma_{z}$ in Proposition 3.1 is the $\gamma$-field associated with the realization $\{A, \Gamma\}$ for $F$, see (2.3). Furthermore, if $\alpha=\infty$, then $\Gamma_{R}$ should be interpreted to be

$$
\Gamma_{R}=\left(\frac{\xi^{*} F\left(z_{0}\right)}{\bar{\beta}-z_{0}} R\left(z_{0}\right) \quad \Gamma R\left(z_{0}\right) \quad-\frac{1}{\beta-z_{0}} \frac{\eta^{*}}{\eta^{*} \xi}\right)^{T},
$$

and if $\beta=\infty$, then $\left\{A_{R}, \Gamma_{R}\right\}$ should be interpreted to be

$$
\left(A_{R}-z\right)^{-1}=\left(\begin{array}{ccc}
0 & \xi^{*} \Gamma_{\bar{z}}^{[*]} & \xi^{*} F(z) \xi \\
0 & (A-z)^{-1} & \Gamma_{z} \xi \\
0 & 0 & 0
\end{array}\right), \quad \Gamma_{R}=\left(\begin{array}{c}
\xi^{*} F\left(z_{0}\right) R\left(z_{0}\right) \\
\Gamma R\left(z_{0}\right) \\
\frac{\eta^{*}}{\eta^{*} \xi}
\end{array}\right)
$$

Proof. Here only the case $\alpha, \beta \in \mathbb{C}$ is treated; the cases $\alpha=\infty$ or $\beta=\infty$ follow by analogous arguments.

First the selfadjointness of $A_{R}$ is established. Therefore let $H(z):=\left(A_{R}-\right.$ $z)^{-1}$. Then the formula in (3.2) shows that $H(z)$ is an everywhere defined operator for $z \in \rho(A) \backslash\{\beta, \bar{\beta}\}$. In particular, since $\rho(A) \neq \emptyset, \rho(A)$ contains all of $\mathbb{C} \backslash \mathbb{R}$ except finitely many points, for all those points $H(z)$ is an everywhere defined bounded 
operator. Moreover, a direct calculation shows that $H(z)^{[*]}=H(\bar{z})$. Next we establish that $H$ satisfies the resolvent identity. Therefore note that a calculation shows that $H(z) H(w)$ is equal to

$$
\left(\begin{array}{ccc}
\frac{1}{(\bar{\beta}-z)(\bar{\beta}-w)} & \frac{\xi^{*}}{\bar{\beta}-z}\left(\frac{\Gamma_{\bar{w}}^{[*]}}{\bar{\beta}-w}+\Gamma_{\bar{z}}^{[*]}(A-w)^{-1}\right) & \xi^{* \frac{F(w)}{\bar{\beta}-w}+\Gamma_{\bar{z}}^{[*]} \Gamma_{w}+\frac{F(z)}{\beta-z}} \\
0 & (A-z)^{-1}(A-w)^{-1} & \left((A-z)^{-1} \Gamma_{w}+\frac{\Gamma_{z}}{\beta-z}\right) \frac{\xi}{\beta-w} \\
0 & 0 & \frac{1}{(\beta-z)(\beta-w)}
\end{array}\right) .
$$

Using (2.3) and the resolvent identity for $A$ we have that

$$
\begin{aligned}
\Gamma_{\bar{z}}^{[*]}(A-w)^{-1} & =\Gamma^{[*]}\left(I+\left(z-\overline{z_{0}}\right)(A-z)^{-1}\right)(A-w)^{-1} \\
& =\Gamma^{[*]}\left((A-w)^{-1}+\frac{z-\overline{z_{0}}}{z-w}\left((A-z)^{-1}-(A-w)^{-1}\right)\right) \\
& =\frac{\Gamma^{[*]}}{z-w}\left(\left(z-\overline{z_{0}}\right)(A-z)^{-1}-\left(w-\overline{z_{0}}\right)(A-w)^{-1}\right)=\frac{\Gamma_{\bar{z}}^{[*]}-\Gamma_{\bar{w}}^{[*]}}{z-w} .
\end{aligned}
$$

Moreover, using (2.4) we have that

$$
\begin{aligned}
\frac{F(w)}{\bar{\beta}-w}+\Gamma_{\bar{z}}^{[*]} \Gamma_{w}+\frac{F(z)}{\beta-z} & =F(z)\left(\frac{1}{\beta-z}+\frac{1}{z-w}\right)+F(w)\left(\frac{1}{\bar{\beta}-w}-\frac{1}{z-w}\right) \\
& =\frac{1}{z-w}\left(\frac{\beta-w}{\beta-z} F(z)-\frac{\bar{\beta}-z}{\bar{\beta}-w} F(w)\right) .
\end{aligned}
$$

Combining the three preceding expressions and using the resolvent identity for $A$ yields that $H(z) H(w)=\frac{H(z)-H(w)}{z-w}$. Consequently, $A_{R}$ is a selfadjoint relation in $\left\{\Pi_{2},[\cdot, \cdot]_{2}\right\}$, see $[4$, Prop. 3.4 and Cor. on p. 162].

As the second step towards proving that $\left\{A_{R}, \Gamma_{R}\right\}$ realizes $F_{R}$, the $\gamma$-field associated with $\left\{A_{R}, \Gamma_{R}\right\}$ is determined. Using

$$
\frac{z_{0}-\bar{\alpha}}{z_{0}-\beta}+\frac{z-z_{0}}{\beta-z} \frac{\bar{\alpha}-\beta}{\beta-z_{0}}=\frac{\bar{\alpha}-\beta}{\beta-z}+1=\frac{z-\bar{\alpha}}{z-\beta}
$$

and the identity $\left(z-z_{0}\right) \Gamma_{\bar{z}}^{[*]} \Gamma=F(z)-F\left(z_{0}\right)$, see $(2.4)$, a straight-forward calculation shows that

$$
\left(\Gamma_{R}\right)_{z}:=\left(I+\left(z-z_{0}\right)\left(A_{R}-z\right)^{-1}\right) \Gamma_{R}=\left(\begin{array}{lll}
\frac{\xi^{*} F(z)}{\bar{\beta}-z} R(z) \quad \Gamma_{z} R(z) \quad \frac{\bar{\alpha}-\beta}{\beta-z} \frac{\eta^{*}}{\eta^{*} \xi}
\end{array}\right)^{\top} .
$$

Combining this last result with (3.3) and the identity $\left(z-\overline{z_{0}}\right) \Gamma^{[*]} \Gamma_{z}=F(z)-F\left(\overline{z_{0}}\right)$ from (2.4) leads to

$$
\begin{aligned}
\left(z-\overline{z_{0}}\right) \Gamma_{R}^{[*]}\left(\Gamma_{R}\right)_{z}= & \frac{z-\overline{z_{0}}}{\bar{\beta}-z} \frac{\alpha-\bar{\beta}}{\bar{\beta}-\overline{z_{0}}} \frac{\eta \xi^{*}}{\xi^{*} \eta} F(z) R(z)+R\left(z_{0}\right)^{*} F\left(\overline{z_{0}}\right) \frac{z-\overline{z_{0}}}{\beta-\bar{\alpha}} \frac{\bar{\alpha}-\beta}{\beta-z} \frac{\xi \eta^{*}}{\eta^{*} \xi} \\
& +\left[\left(I-P^{*}\right)+\frac{\overline{z_{0}}-\alpha}{\overline{z_{0}}-\bar{\beta}} P^{*}\right]\left(F(z)-F\left(\overline{z_{0}}\right)\right)\left[(I-P)+\frac{z-\bar{\alpha}}{z-\beta} P\right] \\
= & R^{\#}(z) F(z) R(z)-R^{\#}\left(\overline{z_{0}}\right) F\left(\overline{z_{0}}\right) R\left(\overline{z_{0}}\right)=F_{R}(z)-F_{R}\left(\overline{z_{0}}\right) .
\end{aligned}
$$


This shows that $\left\{A_{R}, \Gamma_{R}\right\}$ realizes $F_{R}$, see (2.4).

\subsection{Decomposing generalized Nevanlinna functions}

Recall that $\rho(F)$ and $\sigma(F)$ denote the set of holomorphy of a generalized Nevanlinna function $F$ in $\mathbb{C} \cup\{\infty\}$ and its complement, respectively. When $F$ is minimally realized by $\{A, \Gamma\}$, then $\rho(F)$ and $\sigma(F)$ coincide with $\rho(A)$ and $\sigma(A)$, respectively, see Theorem 2.2 .

Proof of Theorem 1.1. Let $z_{0} \in \rho(F) \cap(\mathbb{C} \backslash \mathbb{R})(\neq \emptyset)$, then there exists an everywhere defined selfadjoint operator $C$ in $\mathcal{H}$ such that $\operatorname{ran}\left(F\left(z_{0}\right)+C\right)=\mathcal{H}$, i.e., that $F+C$ is boundedly invertible at $z_{0}$. Since the statement clearly holds for $F$ if it holds for $F+C$, we may w.l.o.g. assume that $F$ is boundedly invertible at a point $z_{0} \in \rho(F) \cap(\mathbb{C} \backslash \mathbb{R})$, cf. [14, Prop. 2.1]; such operator-valued generalized Nevanlinna functions are called regular.

Let $\left\{\alpha_{1}, \ldots, \alpha_{\kappa}\right\}$ and $\left\{\beta_{1}, \ldots, \beta_{\kappa}\right\}$ be the sets of all GPNTs of $F$ and $-F^{-1}$ in $\mathbb{C}_{+} \cup \mathbb{R} \cup\{\infty\}$, respectively; here each GPNT occurs in accordance with its multiplicity. Since $F$ is assumed to be regular, [14, Thm. 5.2 and Cor. 5.3] yield the existence of $\eta_{1}, \xi_{1}, \tilde{\eta}_{1}, \tilde{\xi}_{1} \in \mathcal{H}$ satisfying $\eta_{1}^{*} \xi_{1} \neq 0$ and $\tilde{\eta}_{1}^{*} \tilde{\xi}_{1} \neq 0$ such that $F_{1}:=R_{1}^{\#} F R \in \mathfrak{N}_{\kappa-1}(\mathcal{H})$, where

$$
R_{1}(z)=R\left(z ; \beta_{1}, \bar{\gamma}, \tilde{\eta}_{1}, \tilde{\xi}_{1}\right) R\left(z ; \gamma, \overline{\alpha_{1}}, \eta_{1}, \xi_{1}\right) ;
$$

here $\gamma$ is an arbitrary element of $\mathbb{C} \backslash\left(\mathbb{R} \cup \operatorname{GPNT}(F) \cup \operatorname{GPNT}\left(-F^{-1}\right)\right)$. Moreover, the cited statements yield that $\left\{\alpha_{2}, \ldots, \alpha_{\kappa}\right\}$ and $\left\{\beta_{2}, \ldots, \beta_{\kappa}\right\}$ are the sets of all GPNTs of $F_{1}$ and $-F_{1}^{-1}$ in $\mathbb{C}_{+} \cup \mathbb{R} \cup\{\infty\}$, respectively. Since $F_{1}$ is evidently regular, inductively applying this argument yields that $F$ can be factorized as $R^{\#} F_{0} R$, where $F_{0} \in \mathfrak{N}_{0}(\mathcal{H})$ and

$$
R(z)=\prod_{j=1}^{\kappa} R\left(z ; \beta_{i}, \bar{\gamma}, \tilde{\eta}_{i}, \tilde{\xi}_{i}\right) R\left(z ; \gamma, \overline{\alpha_{i}}, \eta_{i}, \xi_{i}\right) ;
$$

here $\gamma$ is any element of $\mathbb{C} \backslash\left(\mathbb{R} \cup \operatorname{GPNT}(F) \cup \operatorname{GPNT}\left(-F^{-1}\right)\right)$ and $\eta_{i}, \xi_{i}, \tilde{\eta}_{i}, \tilde{\xi}_{i} \in \mathcal{H}$ satisfy $\eta_{i}^{*} \xi_{i} \neq 0 \neq \tilde{\eta}_{i}^{*} \tilde{\xi}_{i}$ for $i=1, \ldots, \kappa$. For later usage introduce the set $\mathcal{P}_{0}$ as

$$
\mathcal{P}_{0}:=\{\gamma, \bar{\gamma}\} \cup \operatorname{GPNT}(F)=\{\gamma, \bar{\gamma}\} \cup\left\{\alpha_{1}, \ldots, \alpha_{\kappa}, \overline{\alpha_{1}}, \ldots, \overline{\alpha_{\kappa}}\right\} .
$$

Let $\left\{A_{0}, \Gamma_{0}\right\}$ realize $F_{0}$ minimally, then the corresponding realizing space is a Hilbert space $\{\mathfrak{H},(\cdot, \cdot)\}$, see e.g. [15]. Using the spectral family of $A_{0}, \mathfrak{H}$ can be decomposed as $\mathfrak{H}_{1} \oplus \mathfrak{H}_{2}$ such that, with $A_{i}$ defined via $\operatorname{gr}\left(A_{i}\right)=\operatorname{gr}(A) \cap\left(\mathfrak{H}_{i} \times \mathfrak{H}_{i}\right)$,

(a) $\left\{\mathfrak{H}_{i},(\cdot, \cdot)\right\}$ is a Hilbert space and $\mathfrak{H}_{i}$ is $A$-invariant for $i=1,2$;

(b) $\sigma\left(A_{1}\right) \subseteq \operatorname{clos} \Delta$ and $\operatorname{int} \Delta \subseteq \rho\left(A_{2}\right)$;

(c) $\sigma_{p}\left(A_{1}\right) \cap \sigma_{p}\left(A_{2}\right)=\emptyset$.

For instance, if $\Delta=(a, b) \subseteq \mathbb{R}$, then the desired decomposition with the properties (a)-(c) can be obtained by taking $\mathfrak{H}_{1}$ to be $E_{b}-E_{a}$, where $\left\{E_{x}\right\}_{x \in \mathbb{R}}$ is the spectral family associated with the Hilbert space selfadjoint relation $A_{0}$. 
Since $\left\{A_{0}, \Gamma_{0}\right\}$ is a minimal realization for $F_{0}$, the decomposition with the properties (a)-(c) induces an additive representation $F_{0}=F_{1}+F_{2}$, where $F_{j}$ is an ordinary Nevanlinna function realized by $\left\{A_{j}, P_{j} \Gamma_{0}\right\}$; here $P_{j}$ is the $\Pi$-orthogonal projection onto $\mathfrak{H}_{j}$ for $j=1,2$, see the discussion following (2.10). Notice that the realizations $\left\{A_{1}, P_{1} \Gamma_{0}\right\}$ and $\left\{A_{2}, P_{2} \Gamma_{0}\right\}$ are automatically minimal, because the realization $\left\{A_{0}, \Gamma_{0}\right\}$ is assumed to be minimal. Inserting this additive representation $F_{0}=F_{1}+F_{2}$ into the factorization $F=R^{\#} F_{0} R$ produces the following decomposition for $F$ :

$$
F(z)=R^{\#}(z) F_{0}(z) R(z)=R^{\#}(z) F_{1} R(z)+R^{\#}(z) F_{2}(z) R(z) .
$$

Next the terms $R^{\#} F_{1} R$ and $R^{\#} F_{2} R$ are considered separately. In order to treat them, divide $\mathcal{P}_{0}$, see (3.5), into the following three sets:

$$
\mathcal{P}_{\Delta}=\mathcal{P}_{0} \cap \operatorname{int} \Delta, \quad \mathcal{P}_{c}=\mathcal{P}_{0} \cap \partial \Delta \quad \text { and } \quad \mathcal{P}_{r}=\mathcal{P}_{0} \backslash\left(\mathcal{P}_{\Delta} \cup \mathcal{P}_{c}\right)
$$

$R^{\#} F_{1} R$ : By Proposition 3.1 there exist an extension $A_{1, R}$ of $A_{1}$ in a Pontryagin space $\left\{\Pi_{1, R},[\cdot, \cdot]_{1, R}\right\}$ (with at most $2 \kappa$ negative squares since, in addition to the poles $\overline{\alpha_{i}}, R$ in (3.4) can have at most $\kappa$ additional poles located at $\bar{\gamma}$ ) and a mapping $\Gamma_{1, R}$ such that $\left\{A_{1, R}, \Gamma_{1, R}\right\}$ realizes $R^{\#} F_{1} R$. Furthermore, Proposition 3.1 shows that

$$
\sigma\left(A_{1, R}\right) \subseteq \sigma\left(A_{1}\right) \cup \mathcal{P}_{0}=\sigma\left(A_{1}\right) \cup \mathcal{P}_{\Delta} \cup \mathcal{P}_{c} \cup \mathcal{P}_{r}
$$

By definition, see (b) and (3.7), $\mathcal{P}_{r}$ consists of (finitely many) isolated points of the spectrum $\sigma\left(A_{1, R}\right)$. Therefore $\left\{\Pi_{1, R},[\cdot, \cdot]\right\}$ can by means of Riesz projections (contour integrals of the resolvent, see e.g. [1, Ch. 2: Thm. 2.20]) be decomposed as $\Pi_{1, R}^{1}[+] \Pi_{1, R}^{2}$, such that, with $A_{1, R, i}$ defined by $\operatorname{gr}\left(A_{1, R, i}\right)=\operatorname{gr}\left(A_{1, R}\right) \cap\left(\Pi_{1, R}^{i} \times\right.$ $\left.\Pi_{1, R}^{i}\right)$, the following statements hold:

(a $\left.\mathrm{a}_{1}\right)\left\{\Pi_{1, R}^{i},[\cdot, \cdot]_{1, R}\right\}$ is a Pontryagin space and $\Pi_{1, R}^{i}$ is $A_{1, R}$-invariant for $i=1,2$;

$\left(\mathrm{b}_{1}\right) \sigma\left(A_{1, R, 1}\right) \subseteq \sigma\left(A_{1}\right) \cup \mathcal{P}_{\Delta} \cup \mathcal{P}_{c} \subseteq \operatorname{clos} \Delta$;

$\left(\mathrm{c}_{1}\right) \sigma\left(A_{1, R, 2}\right) \subseteq \mathcal{P}_{r}$ and, hence, int $\Delta \subseteq \rho\left(A_{1, R, 2}\right)$.

$R^{\#} F_{2} R$ : By Proposition 3.1 there exist an extension $A_{2, R}$ of $A_{2}$ in a Pontryagin space $\left\{\Pi_{2, R},[\cdot, \cdot]_{2, R}\right\}$ (again with at most $2 \kappa$ negative squares) and a mapping $\Gamma_{2, R}$ such that $\left\{A_{2, R}, \Gamma_{2, R}\right\}$ realizes $R^{\#} F_{2} R$. Again Proposition 3.1 shows that

$$
\sigma\left(A_{2, R}\right) \subseteq \sigma\left(A_{2}\right) \cup \mathcal{P}_{0}=\sigma\left(A_{2}\right) \cup \mathcal{P}_{\Delta} \cup \mathcal{P}_{c} \cup \mathcal{P}_{r}
$$

Hence, by construction (see (b)) there exist an open neighborhood $\mathcal{O}$ (in $\mathbb{C}$ ) containing $\mathcal{P}_{\Delta}$ such that $\mathcal{O} \backslash \mathcal{P}_{\Delta} \subseteq \rho\left(A_{2, R}\right)$. Thus $\left\{\Pi_{2, R},[\cdot, \cdot]\right\}$ can by means of Riesz projections (see [1, Ch. 2: Thm. 2.20]) be decomposed as $\Pi_{2, R}^{1}[+] \Pi_{2, R}^{2}$, where, with $A_{2, R, i}$ defined via $\operatorname{gr}\left(A_{2, R, i}\right)=\operatorname{gr}\left(A_{2, R}\right) \cap\left(\Pi_{2, R}^{i} \times \Pi_{2, R}^{i}\right)$, the following statements hold:

(a $\left\{\Pi_{2, R}^{i},[\cdot, \cdot]_{2, R}\right\}$ is a Pontryagin space and $\Pi_{2, R}^{i}$ is $A_{2, R}$-invariant for $i=1,2$;

( $\left.\mathrm{b}_{2}\right) \sigma\left(A_{2, R, 1}\right) \subseteq \mathcal{P}_{\Delta} \subseteq \Delta$

$\left(\mathrm{c}_{2}\right) \sigma\left(A_{2, R, 2}\right) \subseteq \sigma\left(A_{2}\right) \cup \mathcal{P}_{c} \cup \mathcal{P}_{r}$ and, hence, int $\Delta \subseteq \rho\left(A_{2, R, 2}\right)$. 
Now we reconsider $F$ and decompose it as claimed in Theorem 1.1. Therefore let $F_{i, j}$ be the function realized by $\left\{A_{i, R, j}, \Gamma_{i, R, j}\right\}$ for $i, j=1,2$. By means of these functions define $F_{\Delta}$ and $F_{R}$ as

$$
F_{\Delta}:=F_{1,1}+F_{2,1} \quad \text { and } \quad F_{R}:=F_{1,2}+F_{2,2} .
$$

We claim that these functions satisfy all the criteria in Theorem 1.1. Indeed, by construction the functions $F_{\Delta}$ and $F_{R}$ are (possibly non-minimally) realized by $\left\{A_{\Delta}, \Gamma_{\Delta}\right\}:=\left\{A_{1, R, 1} \oplus A_{2, R, 1}, \operatorname{col}\left(\Gamma_{1, R, 1}, \Gamma_{2, R, 1}\right)\right\}$ and $\left\{A_{R}, \Gamma_{R}\right\}:=\left\{A_{1, R, 2} \oplus\right.$ $\left.A_{2, R, 2}, \operatorname{col}\left(\Gamma_{1, R, 2}, \Gamma_{2, R, 2}\right)\right\}$, see Section 2.4. Therefore (b)-(c), (b $\left.\mathrm{b}_{1}\right)-\left(\mathrm{c}_{1}\right)$ and $\left(\mathrm{b}_{2}\right)$ $\left(\mathrm{c}_{2}\right)$ show that

$$
\sigma\left(A_{\Delta}\right) \subseteq \operatorname{clos} \Delta, \quad \operatorname{int}(\Delta) \subseteq \rho\left(A_{R}\right) \quad \text { and } \quad \sigma_{p}\left(A_{\Delta}\right) \cap \sigma_{p}\left(A_{r}\right) \subseteq \mathcal{P}_{c} .
$$

Since $\mathcal{P}_{c}$ is by definition equal to $(\{\gamma, \bar{\gamma}\} \cup \operatorname{GPNT}(F)) \cap \partial \Delta$, cf. (3.5) and (3.7), (2.5) and Proposition 2.3 show that all the assertions in Theorem 1.1 hold except the assertions about the sum of the indices $\kappa_{\Delta}$ and $\kappa_{R}$ of $F_{\Delta}$ and $F_{R}$. The fact that $\kappa_{\Delta}+\kappa_{R} \geq \kappa$ is indicated in the discussion preceding (2.9). The final assertion in Theorem 1.1 that $\kappa_{\Delta}+\kappa_{R}=\kappa$ if $\operatorname{GPNT}(F) \cap(\operatorname{clos}(\Delta) \backslash \Delta)=\emptyset$ is now a consequence of Proposition 2.5.

Inductively applying the preceding statement to the case when $\Delta$ is an interval of $\mathbb{R} \cup\{\infty\}$ containing precisely one GPNT in its interior yields Corollary 3.2 below. Note in connection with Corollary 3.2 that since non-real poles of a generalized Nevanlinna function are isolated, we can always write a generalized Nevanlinna function as the sum of a generalized Nevanlinna function holomorphic in $\mathbb{C} \backslash \mathbb{R}$ with rational functions each having a pole only at a non-real point and its conjugate.

Corollary 3.2. Let $F \in \mathfrak{N}_{\kappa}(\mathcal{H})$ and let $\operatorname{GPNT}(F)=\left\{\alpha_{1}, \ldots, \alpha_{n}, \overline{\alpha_{1}}, \ldots, \overline{\alpha_{n}}\right\}$ where $\alpha_{1}, \ldots, \alpha_{n}$ are distinct elements of $\mathbb{C} \cup\{\infty\}$. Then $F=\sum_{i=1}^{n} F_{i}$, where

(i) $F_{i} \in \mathfrak{N}_{\kappa_{i}}(\mathcal{H})$, for $i=1, \ldots, n$, and $\sum_{i=1}^{n} \kappa_{i}=\kappa$;

(ii) $\operatorname{GPNT}\left(F_{i}\right)=\left\{\alpha_{i}, \overline{\alpha_{i}}\right\}$, for $i=1, \ldots, n$;

(iii) $\sigma\left(F_{i}\right) \cap \sigma\left(F_{j}\right)$ contains at most two points and any point contained in the intersection is not both a generalized pole for $F_{i}$ and $F_{j}$, for $1 \leq i \neq j \leq n$.

\subsection{Decomposing selfadjoint relations in Pontryagin spaces}

In order to prove Theorem 1.2 the result from the preceding section is lifted to the setting of selfadjoint relations by associating to (the resolvent) of selfadjoint relations an (operator-valued) generalized Nevanlinna function.

Proof of Theorem 1.2. Let $J$ be any canonical symmetry for the Pontryagin space $\{\Pi,[\cdot, \cdot]\}$ appearing in Theorem 1.2. Then $\{\mathcal{H},(\cdot, \cdot)\}:=\{\Pi,[J \cdot, \cdot]\}$ defines a Hilbert space, see e.g. [1, Ch. $1, \S 3]$. In addition to the given selfadjoint relation $A$ in the Pontryagin space $\Pi$ introduce the operator $\Gamma: \mathcal{H}(=\Pi) \rightarrow \Pi$ as the identity mapping. Then the pair $\{A, \Gamma\}$ provides a minimal realization for the following generalized Nevanlinna function:

$$
F(z)=\overline{z_{0}} J+\left(z-\overline{z_{0}}\right) J\left(I+\left(z-z_{0}\right)(A-z)^{-1}\right), \quad z_{0}, z \in \rho(A) ;
$$


cf. (2.2). Let $F_{\Delta}+F_{R}$ be the additive decomposition of $F$ provided by Theorem 1.1 with respect to $\Delta$ as in Theorem 1.2. In particular,

$$
\sigma\left(F_{\Delta}\right) \subseteq \operatorname{clos} \Delta \text { and int } \Delta \subseteq \rho\left(F_{R}\right) .
$$

If $\left\{A_{\Delta}, \Gamma_{\Delta}\right\}$ and $\left\{A_{R}, \Gamma_{R}\right\}$ are arbitrary minimal realizations for $F_{\Delta}$ and $F_{R}$, respectively, then $\left\{A_{\Delta} \widehat{\oplus} A_{R}, \operatorname{col}\left(\Gamma_{\Delta}, \Gamma_{R}\right)\right\}$ is a realization for $F$. Moreover, by Theorem $2.2 \sigma\left(A_{\Delta}\right)=\sigma\left(F_{\Delta}\right)$ and $\rho\left(A_{R}\right)=\rho\left(F_{R}\right)$. In view of (3.9), the first statement in Theorem 1.2 now holds by Proposition 2.3 and 2.1 .

Finally, if $\partial \Delta \cap \operatorname{ENT}(A)=\emptyset$, then by definition $\partial \Delta \cap \operatorname{GPNT}(F)=\emptyset$. Thus the additive decomposition $F_{\Delta}+F_{R}$ of $F$ with respect to $\Delta$ provided by Theorem 1.1 has the following properties:

(a) $\sigma\left(F_{\Delta}\right) \subseteq \operatorname{clos} \Delta$ and int $\Delta \subseteq \rho\left(F_{R}\right)$;

(b) no point of $\operatorname{clos}(\Delta) \backslash \Delta$ is both a generalized pole of $F_{\Delta}$ and $F_{R}$.

Let $\left\{A_{\Delta}, \Gamma_{\Delta}\right\}$ and $\left\{A_{R}, \Gamma_{R}\right\}$ be arbitrary minimal realizations for the function $F_{\Delta}$ and $F_{R}$, respectively. By Theorem 2.2 and the definition of generalized poles (see Section 2.2) the preceding two properties imply that

(a') $\sigma\left(A_{\Delta}\right) \subseteq \operatorname{clos} \Delta$ and int $\Delta \subseteq \rho\left(A_{R}\right)$;

(b') $\sigma_{p}\left(A_{\Delta}\right) \cap \sigma_{p}\left(A_{R}\right)=\emptyset$.

Thus Proposition 2.6 implies that $\left\{A_{\Delta} \widehat{\oplus} A_{R}, \operatorname{col}\left(\Gamma_{\Delta}, \Gamma_{R}\right)\right\}$, see (2.9), is a minimal realization for $F$ in (3.8). Therefore the statement has been proven, because all minimal realizations for the same generalized Nevanlinna function are unitarily equivalent by Proposition 2.1.

The assumption $\rho(A) \neq \emptyset$ in Theorem 1.2 is needed, because there exist selfadjoint relations $A$ (even in finite-dimensional) Pontryagin spaces for which $\sigma_{p}(A)=\mathbb{C} \cup\{\infty\} ;$ see $[4$, p. $155-156]$.

Applying Theorem 1.2 inductively leads to the following decomposition results for selfadjoint relations. Note that from Corollary 3.3 the canonical form of selfadjoint operators in finite-dimensional Pontryagin spaces, see [5, Thm. 5.1.1.], can be derived.

Corollary 3.3. Let $A$ be a selfadjoint relation in a Pontryagin space $\{\Pi,[\cdot, \cdot]\}$ with $\sigma(A) \cap\left(\mathbb{C}_{+} \cup \mathbb{R} \cup\{\infty\}\right)=\left\{\alpha_{1}, \ldots, \alpha_{n}\right\}$. Then there exists a decomposition $\Pi_{1}[+] \ldots[+] \Pi_{n}$ of $\Pi$ such that

(i) $\left\{\Pi_{i},[\cdot, \cdot]\right\}$ is a Pontryagin space for $i=1, \ldots, n$;

(ii) $\Pi_{i}$ is A-invariant for $i=1, \ldots, n$;

(iii) $\sigma\left(A \prod_{\Pi_{i}}\right)=\left\{\alpha_{i}, \overline{\alpha_{i}}\right\}$ for $i=1, \ldots, n$.

Corollary 3.4. Let $A$ be a selfadjoint relation in a Pontryagin space $\{\Pi,[\cdot, \cdot]\}$ with $\rho(A) \neq \emptyset$ and let $\operatorname{ENT}(A)=\left\{\alpha_{1}, \ldots, \alpha_{n}, \overline{\alpha_{1}}, \ldots, \overline{\alpha_{n}}\right\}$. Then there exists a decomposition $\Pi_{1}[+] \ldots[+] \Pi_{n}$ of $\Pi$ such that

(i) $\left\{\Pi_{i},[\cdot, \cdot]\right\}$ is a Pontryagin space for $i=1, \ldots, n$; 
(ii) $\Pi_{i}$ is $A$-invariant for $i=1, \ldots, n$;

(iii) $\left\{\alpha_{1}, \ldots, \alpha_{i-1}, \alpha_{i+1}, \ldots \alpha_{n}\right\} \in \rho\left(A \uparrow_{\Pi_{i}}\right)$ for $i=1, \ldots, n$;

(iv) $\sigma_{p}\left(A\left\lceil_{\Pi_{i}}\right) \cap \sigma_{p}\left(A\left\lceil_{\Pi_{j}}\right)=\emptyset\right.\right.$ and $\sigma\left(A\left\lceil_{\Pi_{i}}\right) \cap \sigma\left(A\left\lceil_{\Pi_{j}}\right)\right.\right.$ contains at most finitely many points, for $1 \leq i \neq j \leq n$.

Observe that condition (iii) in Corollary 3.4 implies that $\alpha_{i}$ and $\overline{\alpha_{i}}$ are the only ENTs of $A$ restricted to $\Pi_{i}$ for $i=1, \ldots, n$.

\section{References}

[1] T. Ya. Azizov and I. S. Iokhvidov, Linear operators in spaces with an indefinite metric, John Wiley and Sons, New York, 1989.

[2] K. Daho, and H. Langer, "Matrix function of the class $\mathfrak{N}_{\kappa}$ ", Math. Nachr., 120 (1985), 275-294.

[3] A. Dijksma, H. Langer, and H.S.V. de Snoo, "Eigenvalues and pole functions of Hamiltonian systems with eigenvalue depending boundary conditions", Math. Nachr., 161 (1993), 107-154.

[4] A. Dijksma and H. S. V. de Snoo, "Symmetric and selfadjoint relations in Kreĭn spaces I", Oper. Theory Adv. Appl., 24 (1987), 145-166.

[5] I. Gohberg, P. Lancaster, and L. Rodman, Indefinite linear algebra and applications, Birkhäuser Verlag, Basel, 2005.

[6] S. Hassi, M. Kaltenbäck, and H.S.V. de Snoo, "The sum of matrix Nevanlinna functions and selfadjoint extensions in exit spaces", Oper. Theory Adv. Appl., 103 (1998), $137-154$

[7] S. Hassi, H.S.V. de Snoo, and H. Woracek, "Some interpolation problems of Nevanlinna-Pick type", Oper. Theory Adv. Appl., 106 (1998), 201-216.

[8] S. Hassi and H.L. Wietsma, "Products of generalized Nevanlinna functions with symmetric rational functions", J. Funct. Anal. 266 (2014), 3321-3376.

[9] I.S. Iohvidov, M.G. KreĬn and H. Langer, Introduction to the spectral theory of operators in spaces with an indefinite metric, Akademie-Verlag, Berlin, 1982.

[10] P. Jonas, "Operator representations of definitizable functions", Ann. Acad. Sci. Fenn. Math. 25 (2000), 41-72.

[11] M.G. Kreı̆n and H. Langer, "Über die Q-Funktion eines $\pi$-hermiteschen Operators im Raume $\Pi_{\kappa} "$, Acta Sci. Math. (Szeged) 34 (1973), 191-230.

[12] M.G. Kreı̆n and H. Langer, "Über einige Fortsetzungsprobleme, die eng mit der Theorie hermitescher Operatoren im Raum $\Pi_{\kappa}$ zusammenhängen, I. Einige Funktionenklassen und ihre Darstellungen", Math. Nachr. 77 (1977), 187-236.

[13] H. Langer, Spectral functions of definitizable operators in Krĕ̌n spaces, Functional analysis, Proceedings, Dubrovnik 1981, Lecture Notes in Mathematics 948, Berlin 1982.

[14] A. Luger, "A factorization of regular generalized Nevanlinna functions", Integral Equations Operator Theory 43 (2002), 326-345.

[15] H. Langer and B. Textorius, "On generalized resolvents and $Q$-functions of symmetric linear relations (subspaces) in Hilbert space", Pacific J. of Math., 72 (1977), 135-165. 
[16] H.L. Wietsma, "Factorization of generalized Nevanlinna functions and the invariant subspace property", Indagationes Mathematicae 30 (2019), 26-38.

Seppo Hassi \& Hendrik Luit Wietsma

Department of Mathematics and Statistics

University of Vaasa

P.O. Box 700, 65101 Vaasa

Finland

e-mail: Seppo.Hassi@uwasa.fi \& Rudi.Wietsma@uwasa.fi 